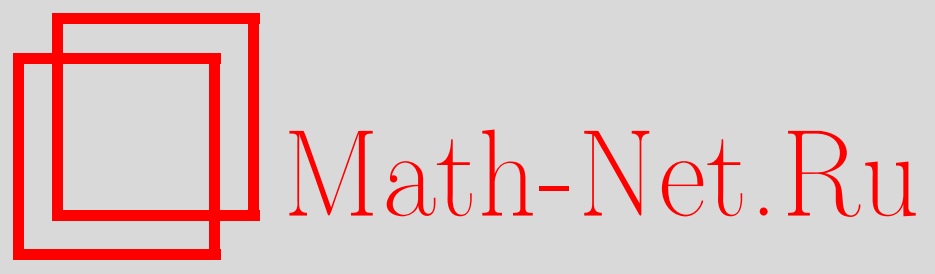

А. А. Черняк, Ж. А. Черняк, Надежность бинарных систем, Дискрет. матем., 1999, том 11, выпуск 1, 129-139

DOI: https://doi.org/10.4213/dm360

Использование Общероссийского математического портала Math-Net.Ru подразумевает, что вы прочитали и согласны с пользовательским соглашением http://www.mathnet.ru/rus/agreement

Параметры загрузки:

IP : 52.205 .19 .152

26 апреля 2023 г., 16:30:19

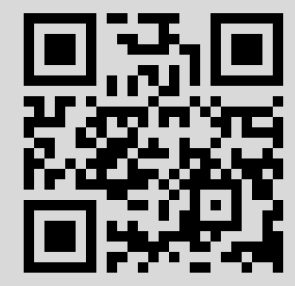




\title{
Надежность бинарных систем
}

\author{
(C) 1999 г. А. А. Черняк, Ж. А. Черняк
}

\begin{abstract}
Основным результатом статьи является теорема, раскрывающая комбинаторную структуру путей и сечений регулярных бинарных систем. В качестве ее прямых следствий получены следующие результаты: даны эффективные алгоритмы определения надежности и минимальных сечений регулярных и униформных регулярных систем; получена конструктивная характеризация регулярных систем, являющихся матроидами; получены эффективно вычисляемые нижние оценки надежности и коэффициентов полинома надежности униформных систем, достигаемые на регулярных системах.
\end{abstract}

\section{1. Введение}

Пусть $T=\{1, \ldots, n\}-$ множество элементов, каждый из которых может находиться в состоянии исправности или отказа, причем элемент $i$ из $T$ подвержен случайному (независимому от других элементов) отказу с вероятностью $1-p, i=1, \ldots, n$. Система $T$, в целом, также может находиться в состоянии отказа или исправности, которое опреджеляется состояниями ее элементов. Подмножество $S \subseteq T$ называется путем (сечением) если исправность (отказ) всех элементов из $S$ влечет исправность (отказ) системы. При этом любое подмножество, содержащее $S$, также является путем (сечением). Последнее свойство означает монотонность системы.

Если $\mathscr{P}=\left\{P_{1}, \ldots, P_{m}\right\}-$ множество всех минимальных (по включению) путей, то пара $[T, \mathscr{P}]$ называется монотонной бинарной системой, а ее надежность $h([T, \mathscr{P}], \bar{p})$, где $\bar{p}=\left(p_{1}, \ldots, p_{n}\right)$, определяется как вероятность наличия в системе хотя бы одного минимального пути, состоящего из исправных элементов. Если мощности всех минимальных путей из $\mathscr{P}$ равны $r$, то $[T, \mathscr{P}]$ называется $r$-униформной системой.

Возможно также двойственное задание монотонной системы парой $[T, \mathscr{R}]$, где $\mathscr{R}=\left\{R_{1}, \ldots, R_{s}\right\}$ - множество минимальных сечений. При этом надежность системы равна единице минус вероятность наличия хотя бы одного сечения, состоящего из отказавших элементов. Отметим, что существуют эквивалентные формулировки понятия монотонных бинарных систем, использующие понятия гиперграфа и булевой функции.

Проблема вычисления надежности монотонной бинарной системы является алгоритмически трудной: доказано, что она остается NP-полной даже в классе 2-униформных систем ограниченной плотности [1-4]. Ввиду этого факта актуальны усилия, направленные на нахождение достаточно представительных классов бинарных систем, надежность которых полиномиально вычислима. Не менее важной задачей

5 Дискретная математика, т.11 №1 
является поиск эффективно вычислимых оценок надежности и коэффициентов полинома надежности униформных систем [5-6].

Хорошо известен класс так называемых шелленговых систем, в котором проблема вчисления надежности полиномиально разрешима $[1,7]$. Интерес к нему обусловлен прежде всего тем, что шелленговыми являются такие комбинаторные структуры, как матроидальные и регулярные системы, играющие важную роль в пороговой логике и целочисленном программировании (см., например, [8-11]).

В данной статье рассматриваются собственно регулярные системы, независимо от свойств более общих классов систем. Основной результат - это теорема 1, характеризующая комбинаторную структуру путей и сечений регулярной системы. Универсальность теоремы 1 раскрывается в следствиях 1-7, в которых , в частности, содержатся следующие результаты.

Получен алгоритм, вычисляющий с временной сложностью $O\left(m+n\left|\Omega^{*}\right|\right)$ надежность и коэффициенты полинома надежности регулярной системы, где $\Omega^{*}$ определяется в следующем параграфе.

Доказано, что за время $O\left(m+n\left|\Omega^{*}\right|\right)$ и $O(m)$ соответственно можно определить множество минимальных сечений регулярной и $r$-униформной регулярной системы. Получена верхняя оценка числа минимальных сечений регулярной системы, заданной совокупностью своих мнимальных путей. Эти результаты усиливают соответствующие результаты, полученные в $[11,12]$.

Получена конструктивная характеризация регулярных систем, являющихся матроидом. В отличие от известного критерия из [9], такая характеризация позволяет эффективно генерировать системы с такими свойствами.

Пороговые и мультипороговые графы являются срециальными случаями униформных регулярных систем. Именно в этих классах графов достигаются нижние оценки надежности. Более точно, в [13] было показано, что существует последовательность преобразований сдвига $[14,15]$, приводящая к пороговому графу, доставляющему эффективно вычисляемую оценку полинома надежности исходного графа.

В данной статье понятие преобразования сдвига обобщено на классы униформных систем. Получены эффективно вычисляемые нижние оценки надежности униформной системы, достигаемые на регулярных системах.

\section{2. Основные определения}

Характеристическим вектором подмножества $A \subseteq T$ называется такой $(0,1) n$-вектор $x=\left(x_{1}, \ldots, x_{n}\right)$, что $\left(x_{i}=1\right) \Longleftrightarrow \quad(i \in A)$. При этом $\operatorname{ind}(x)$ обозначает множество $\left\{i: x_{i}=1\right\}$.

Пусть $\Omega=\left\{\bar{u}_{1}, \ldots, \bar{u}_{m}\right\}-$ множество характеристических векторов минимальных путей системы $[T, \mathscr{P}]$. Положим $f(x)=1$, если $x \geqslant \bar{u}_{i}$ для некоторого $\bar{u}_{i} \in \Omega$, и $f(x)=0$ в противном случае. Функция $f$ называется структурной функцией системы $[T, \Omega]$ (всюду в дальнейшем вместо символа $\mathscr{P}$ используется символ $\Omega$ ). Вектор $x$ называется вектором исправного (отказового) состояния системы $[T, \Omega]$, если $f(x)=1$ $(f(x)=0)$. Для краткости такие векторы будем обозначать РВ и ОВ соответственно.

Скажем, что $x$ является левым сдвигом вектора $y$ (или $y$ является правым сдвигом вектора $x)$, если ind $(x)=\left\{i_{1}, \ldots, i_{r}\right\}$, ind $(y)=\left\{j_{1}, \ldots, j_{r}\right\}, i_{1} \leqslant j_{1}, \ldots, i_{r} \leqslant j_{r}$. Система $[T, \Omega]$ называется регулярной, если множество всех РВ замкнуто относительно левых сдвигов. Система $[T, \Omega]$ является матроидом, если совокупность под- 
множеств, характеристическими векторами которых являются ОВ, удовлетворяет аксиомам независимости матроида.

Вектор $x$ называется максимальным ОВ, если любой вектор $y$ такой, что $y>x$, является РВ.

Введем дополнительные обозначения. Пусть $e_{i}$ - характеристический вектор одноэлементного множества $\{i\} ; \oplus$ обозначает операцию булева сложения;

$$
\begin{aligned}
& x^{*}=x-e_{m(x)}, \\
& m(x)= \begin{cases}\max \{i: i \in \operatorname{ind}(x)\}, & \text { если } \operatorname{ind}(x) \neq \varnothing, \\
0, & \text { если } \operatorname{ind}(x)=\varnothing,\end{cases} \\
& \operatorname{ind}(x, i)=\{j: j \in \operatorname{ind}(x), j>i\}, \\
& j(x \oplus y)=\min \{i: i \in \operatorname{ind}(x \oplus y)\} \text {, } \\
& \operatorname{sh}(x)= \begin{cases}x^{*}+e_{m(x)+1}, & \text { если } m(x) \neq n, \\
x^{*}-e_{m\left(x^{*}\right)}, & \text { если } m(x)=n, x \neq e_{n},\end{cases} \\
& (x ; y)=\{z: x \ll z \ll y\}, \\
& {[x ; y]=(x ; y) \cup\{x, y\} \text {, }} \\
& t(x)=\left\{\begin{array}{l}
\max \left\{i: x_{i}=1, \operatorname{ind}(x, i)<m(x)-i\right\}, \\
0, \text { если таких } i \text { не существует. }
\end{array}\right.
\end{aligned}
$$

Отметим, что ind $(x, i) \leqslant m(x)-i$ и « означает отношение линейного порядка.

Вектор $x^{*}$ называется первой производной вектора $x$. Результат последовательного применения $k$ операций $*$ к $x$ называется $k$-й производной вектора $x$. Сам вектор $x$ считается нулевой производной $x$.

Пусть $y$ есть $(k-1)$-я производная $x$ и $s=m(y)$. Тогда вектор $y^{*}+e_{s+1}+\ldots+e_{n}$ называется замыканием вектора $x$ и обозначается $x[s]\left(\right.$ если $s=n$, то $\left.x[s]=y^{*}\right)$. Отметим, что $x$ имеет ind $(x)$ замыканий.

Для данжого вектора $x$ обозначим через $\Phi(x)$ вектор $y$ из $\Omega$ такой, что $x=y^{*}$ и $y$ имеет максимальную величину $m(y)$. Вектор $\Phi(x)$ будем называть первообразной вектора $x$.

Позиционным представлением вектора $x$ называется $n$-вектор, компонентами которого являются элементы множества ind $(x)$, упорядоченные по возраста̇нию и дополненные в конце нулями. Будем предполагать, что линейный порядок на множестве $(0,1) n$-векторов индуцируется лексикографическим порядком их позиционных представлений.

Через $\Phi^{*}$ обозначим множество всех первых производных векторов из $\Omega$, упорядоченное строго по возрастанию относительно порядка «. Добавим так называемый фиктивный вектор $d$, следующий за всеми $(0,1) n$-векторами (фиктивный вектор будем считать ОВ).

Для вектора вероя́тностей $\bar{p}=\left(p_{1}, \ldots, p_{n}\right)$ обозначим через $\mathbf{P}(x \mid i \leqslant k)$ произведение $p\left(x_{1}\right) \ldots p\left(x_{k}\right)$, где для $i=1, \ldots, n$

$$
p\left(x_{i}\right)= \begin{cases}p_{i}, & \text { если } x_{i}=1, \\ 1-p_{i}, & \text { если } x_{i}=0 .\end{cases}
$$

Величина $\mathbf{P}(x \mid i \leqslant n)$ называется вероятностным весом вектора $x$. Надежность $h([T, \Omega], \bar{p})$ системы $[T, \Omega]$ есть сумма вероятностных весов всех РВ функции $f$. 
Пусть $h_{i}$ - число PB $x$ таких, что $|\operatorname{ind}(x)|=i$, и пусть $p_{1}=\ldots=p_{n}=p$. Тогда выражение $\sum_{i=1}^{n} h_{i} p^{i}\left(1-p_{i}\right)^{n-i}$, равное величине $h([T, \Omega], p)$, называется полиномом надежности с коэффициентами $h_{i}, i=1, \ldots, n$.

\section{3. Регулярные системы}

Теорема 1. Пусть $[T, \Omega]-$ регулярная система, $v$ u $w$ - два последователъных вектора из $\Omega^{*} \cup\{d\}, u=\Phi(x)$. Тогда все векторы сегмента $A=(v, \operatorname{sh}(u))$ являются $P B$, а все векторы сегмента $B=(\operatorname{sh}(u), w)$ являются $О В$. Кроме того, $u \in A$.

Прежде чем перейти к доказательству теоремы, сформулируем и докажем три свойства порядка $\ll$.

Свойство 1. Пусть $k=j(x \oplus y)$. Тогда $x \ll y$, тогда и только тогда, когда

$$
x_{k}=1, \quad y_{k}=0, \quad m(y)>k
$$

или

$$
x_{k}=0, \quad y_{k}=1, \quad m(x)<k \text {. }
$$

Это свойство прямо следует из определения порядка «.

Свойство 2. Если вектор $x$ является $\mathrm{PB}$, а вектор $x^{*}$ является $\mathrm{OB}$, то $x \in \Omega$.

Доказательство. Предположим противное. Пусть существует вектор $y$, являющийся $\mathrm{PB}$, такой, что $y<x$. Тогда $m(y)=m(x)$, ибо иначе $y \leqslant x^{*}$. Отсюда следует, что $y_{i}=0$ и $x=1$ для некоторого $i<m(x)$. В этом случае $z=y^{*}+e_{i} \leqslant x^{*}$. Но $z$ является левым сдвигом $y$, то есть $z$ является $\mathrm{PB}$, что противоречит последнему неравенству.

Свойство 3. Пусть $x=z^{*}$ и $x \ll y \ll z$. Тогда $m(x)<j(x \oplus y)<m(z)$.

Доказательство. Предположим, что $k=j(x \oplus y) \geqslant m(z)$. Если $k=m(z)$, тогда $y_{k}=1$, ввиду того, что $x_{m(z)}=0$. Отсюда следует, что $j(x \oplus y)>m(z)$ и $y_{j(x \oplus y)}=1$. Но в этом случае $m(z)>j(x \oplus y)$ по свойству 1 , примененному к $y \ll z$. Получаем противоречие. Если $k>m(z)$, то $y_{m(z)}=0$. Отсюда следует, что $j(y \oplus z)=m(z)$. Но в этом случае, по свойству $1, m(y) \geqslant k>j(y \oplus z)>m(y)$, что невозможно.

Предположим, что $k \leqslant m(x)$. Тогда $x_{k}-1, y_{k}=0, m(y)>k$ по свойству 1 , примененному к $x \ll y$, и $z_{k}=1, j(y \oplus z)=k$. Следовательно, по свойству 1 , примененному к $y \ll z, m(y)<k$. Получаем противоречие.

Доказательство теоремъ 1. Перейдем к доказательству теоремы. Рассмотрим произвольный вектор $x$ из $(v, u)$. Пусть $k=j(v \oplus x)$. Вначале предположим, что $x \in A$ и $x \neq u$. Если $m(u)=n$, то $(u, \operatorname{sh}(u))=\varnothing$, то есть $x \in(v, u)$. Если $m(u) \neq n$, то $(\operatorname{sh}(u))^{*}=u^{*}=\dot{v}$. В любом случае, по свойству $3, m(v)<k \leqslant m(u)$, что влечет включение ind $(v) \cup\{k\} \subseteq$ ind $(x)$.

Зададим теперь вектор $y$ так, что ind $(y)=\operatorname{ind}(v) \cup\{k\}$. Тогда или $y$ совпадает с $u$, или является его левым сдвигом. Ввиду регулярности $[T, \Omega]$ вектор $y$ является РВ. Следовательно, $x$ - также РВ, ибо $y \leqslant x$.

Рассмотрим вектор $\operatorname{sh}(u)$. Если $m(u) \doteq=n$, то $\operatorname{sh}(u)$ является правым сдвигом $v$, то есть $\operatorname{sh}(u)$ есть ОВ. Пусть $m(u)<n$. Тогда $m(\operatorname{sh}(u))>m(u)$ и $(\operatorname{sh}(u))^{*}=v$. Отсюда, 
по определению первообразной, $\operatorname{sh}(u) \notin \Omega$. По свойству 2 вектор $\operatorname{sh}(u)$ не является OB.

Сегмент $A$, как показано выше, состоит из $\mathrm{PB}$, поэтому $w \notin A, u \in A$,

Предположим теперь, что $x \in B$ и $x$ является РВ.. Обозначим через $y$ вектор, являющийся $\mathrm{PB}$, такой, что $y$ получается из $x$ последовательностью операций $*$, и $y^{*}$ есть ОВ (возможно, $y=x$ ). В силу свойства $2 y \in \Omega, y^{*} \in \Omega^{*}$. Кроме того, согласно свойству $1, y^{*} \ll y \ll x \ll w$. Если $v_{k}=0$, то $m(v)<k$ согласно свойству 1 , примененному к $v \ll x$. Но тогда либо $v=y^{*}$ и в этом случае $y^{*}$ есть правый сдвиг вектора $\operatorname{sh}(u)$, являющегося $\mathrm{OB}$, что невозможно, либо $v \ll y^{*}$ по свойству 1 , и в этим случае $y^{*} \in(v, w)$, что противоречит предположению теоремы. Таким образом, $v_{k}=1$ и для некоторого $r$, согласно свойству $1, x_{r}=1, x_{i}=0, i=k, k+1, \ldots, r-1$.

Если $m(y)=r$, то $z=y^{*}+e_{k} \leqslant v$ и вектор $z$ есть левый сдвиг вектора $y$, являющегося РВ. Следовательно, $v$ является $\mathrm{PB}$, что невозможно. Если $m(y)>r$, то $y_{r}^{*}=1, j\left(y^{*} \oplus v\right)=k, y_{k}^{*}=0$. По свойству $1, v \ll y^{*}$, то есть $y^{*} \in(v, w)$. Это противоречит предположению теоремы.

Ниже приводится алгоритм R-REG, вычисляющий надежность $h([T, \Omega], \bar{p})$ регулярной системы $[T, \Omega]$ и коэффициенты $h_{i}, i=1, \ldots, n$, полинома надежности. Как обычно, $\left(\begin{array}{l}s \\ t\end{array}\right)$ означает биномиальный коэффициент, или число сочетаний из $s$ по $t$, причем $\left(\begin{array}{l}s \\ t\end{array}\right)=0$, если $t>s$.

Алгоритм R-REG. Шаг 1. Для данной регулярной системы $T, \Omega]$ образовать множество $\Omega^{*}$ первых производных векторов из $\Omega$ и упорядочить его по возрастанию относительно «.

Шar 2. $h:=0$;

$$
\begin{aligned}
& \text { for } i=1, \ldots, n \text { do } h_{i}:=0 ; \\
& \text { while } \Omega^{*} \neq \varnothing \text { do } v:=\min \left\{x: x \in \Omega^{*}\right\} \\
& u:=\Phi(v) ; \\
& h:=h+\mathbf{P}(v \mid i \leqslant m(v))-\mathbf{P}(v \mid i \leqslant m(u)) ; \\
& \text { for } t=|\operatorname{ind}(v)|+1, \ldots, n \text { do } k:=t-|\operatorname{ind}(v)| ; \\
& s:=n-m(v) ; \\
& l:=n-m(u) ; \\
& h_{t}:=h_{t}+\left(\begin{array}{l}
s \\
k
\end{array}\right)-\left(\begin{array}{l}
l \\
k
\end{array}\right) ; \\
& \Omega^{*}:=\Omega^{*} \backslash\{v\} ; \\
& \text { end. }
\end{aligned}
$$

Следствие 1. Алгоритм R-REG вычисляет надежность и полином надежности регулярной системы $[T, \Omega]$ за время $O\left(m+n\left|\Omega^{*}\right|\right)$.

Доказательство. Время выполнения шага 1 алгоритма есть $O\left(m+n\left|\Omega^{*}\right|\right)$ (см. [16]). Время выполнения шага 2 есть $O\left(n\left|\Omega^{*}\right|\right)$. Величина $\mathbf{P}(v \mid i \leqslant m(v))$ есть сумма вероятностных весов всех векторов из сегмента $[v, \operatorname{sh}(v))$. Величина $\mathbf{P}(v \mid i \leqslant m(u))$ есть сумма вероятностных весов всех векторов из сегмента $\{v\} \cup[\operatorname{sh}(u), \operatorname{sh}(v))$. Отметим, что если $m(u)=n$, то $\operatorname{sh}(u)=\operatorname{sh}(v)$ и $[\operatorname{sh}(u), \operatorname{sh}(v))=\varnothing$. Следовательно, разность между этими величинами есть сумма вероятностных весов всех векторов из сегмента $(v, \operatorname{sh}(u))$. Остальное следует из теоремы 1 . 
Следствие 2. Пусть $[T, \Omega]-$ регулярная система, $v$ и $w$-два последователънъх вектора из $\Omega^{*} \cup\{d\}, u=\Phi(v), x \in[v, w)$,

$$
k= \begin{cases}j(v \oplus w), & w \neq d \\ 0, & w=d\end{cases}
$$

Тогда $x$ является максималъным ОВ, тогда и толъко тогда, когда $x=u[s]$ для некоторого $s>k$.

Доказательство. Докажем достаточность. Пусть $x=u[s], s>k$. Если $s=n$, то $x=v$. Если $s \neq n$, то $x \in[\operatorname{sh}(u), w)$ по определению порядка $\ll$, и в этом случае $x$ также является OB по теореме 1.

Предположим, что существует вектор $y$, являющийся $\mathrm{OB}$, такой, что $x<y$. Без ограничения общности можно считать, что $y-x=e_{r}$. Тогда $r \leqslant s$ по определению замыкания. Положим $z=x+e_{s}$. Вектор $z$ является правым сдвигом $y$, то есть является ОВ. Но $u \leqslant z$. Получаем противоречие.

Докажем необходимость. Предположим, что $x$ является максимальным ОВ. Если $m(u)=n$, то $v=u[n]$. Если $m(u)<n$, то $v<u[m(u))$ и, следовательно, $v$ не является максимальным ОВ. Поэтому в силу теоремы 1 либо $x=v=u[n]$ и тогда утверждение доказано, либо $x \in[\operatorname{sh}(u), w)$.

Заметим, что ситуация $v<w$ невозможна, ибо $v, w \in \Omega^{*}$. Поэтому в силу теоремы $1 v_{k}=1, w_{k}=0, m(w)>k$. Кроме того, $w_{k+1}=w_{k+2}=\ldots=w_{m(w)}$ ввиду отсутствия векторов из $\Omega^{*}$ в сегменте $(v, w)$.

Положим $l=|\operatorname{ind}(u, k)|, \bar{a}_{0}=u, \bar{a}_{s}=\bar{a}_{s-1}^{*}, s=1, \ldots, l$. Тогда по свойству 1 (при $m(u)=n, s=1, \ldots, l-1)$

$$
\begin{aligned}
\operatorname{sh}\left(\bar{a}_{s}\right) & \ll u\left[m\left(\bar{a}_{s}\right)\right] \leqslant \operatorname{sh}\left(\bar{a}_{s+1}\right), \quad s=1, \ldots, l-1, \\
\operatorname{sh}\left(\bar{a}_{l}\right) & \ll w .
\end{aligned}
$$

Очевидно, что $y \leqslant u\left[m\left(\bar{a}_{s}\right)\right]$ для любого $y$ из $\left[\operatorname{sh}\left(\bar{a}_{s}\right), \operatorname{sh}\left(\bar{a}_{s=1}\right)\right)$ и $y<w$ для любого $y$ из $\left[\operatorname{sh}\left(\bar{a}_{l}\right), w\right)$. Но

$$
[\operatorname{sh}(u), w)=\bigcup_{s=0}^{l-1}\left[\operatorname{sh}\left(\bar{a}_{s}\right), \operatorname{sh}\left(\bar{a}_{s+1}\right)\right) \cup\left[\operatorname{sh}\left(\bar{a}_{l}\right), w\right)
$$

Поэтому, ввиду максимальности $x, x=u[s]$ для некоторого $s>k$.

Очевидно, что $x$ является максимальным ОВ тогда и только тогда, когда вектор $(1, \ldots, 1) \oplus x$ является характеристическим вектором минимального сечения системы $[T, \mathscr{P}]$. Поэтому из следствия 2 вытекают следующие утверждения.

Следствие 3. Число минималъный сечений регулярной системы $[T, \Omega]$ не превосходит $1+\sum_{x \in \Omega^{*}}|\operatorname{ind}(x)|$, причем эта оченнка достижима при каждом значении $\left|\Omega^{*}\right|$.

Доказательство. Сама оценка прямо вытекает из следствия 2. Достижимость этой оценки при произвольном значении $\left|\Omega^{*}\right|$ следует из следующего примера 2-униформной регулярной системы. 
Пусть $T=\{1,2, \ldots, 2 k\}, \mathscr{P}$ состоит из всех таких минимальный путей $\{i, j\}$, для которых $i<j, i \leqslant k, i+j \leqslant 2 k+1$. Очевидно, $\left|\Omega^{*}\right|=k$, а из следствия 2 вытекает, что число минимальных сечений системы $[T, \mathscr{P}]$ равно

$$
k+1=1+\sum_{x \in \Omega^{*}}|\operatorname{ind}(x)|
$$

Следствие 4. Минималъные сечения регулярной системы $[T, \Omega]$ могут быть определены за время $O\left(m+n\left|\Omega^{*}\right|\right)$.

Замечание 1. Следствие 4 усиливает соответствующие результаты из $[11,12]$, где временная сложность приведенных алгоритмов равна $O\left(n^{3} \mathrm{~m}\right)$.

Следствие 5. Минималъные сечения $r$-униформной реаулярной системы $[T, \Omega]$ могут быть определены за время $O(m)$.

Следующая очевидная лемма является переформулировкой аксиом баз матроида.

Лемма 1. Система $[T, \Omega]$ явялется матроидом тогда и только тогда, когда для любых двух максималъных $О В$ х и у выполняются условия $|\operatorname{ind}(x)|=|\operatorname{ind}(y)|$; $\partial л я$ любого $i \in \operatorname{ind}(x) \cap \operatorname{ind}(x \oplus y)$ существует такое $j \in \operatorname{ind}(y) \cap \operatorname{ind}(x \oplus y)$, что вектор $x-e_{i}+e_{j}$ является $O B$.

В дальнейшем $a$ обозначает первый элемент в $\Omega^{*}, q=m(\Phi(a))$. Так как РВ инвариантны относительно левых сдвигов, $\operatorname{ind}(a)=\{1, \ldots, p\}, p<q$.

Следствие 6. Регулярная система $[T, \Omega]$ является матроидом тогда и толъко тогда, когда для каждого $v \in \Omega^{*}$

$$
t(u) \leqslant k, \quad m(u) \leqslant q, \quad|\operatorname{ind}(u)|=p-q+1+m(u),
$$

где $u=\Phi(v), k$ то же, что и в условии следствия 2 .

Доказателъство. Докажем необходимость. В силу следствия 2 векторы $x=u[m(u)]$, $b=\Phi(a)[q]$ являются максимальными ОВ. Поэтому по лемме 1

$$
p+n-q=|\operatorname{ind}(b)|=|\operatorname{ind}(x)|=|\operatorname{ind}(u)|-1+n-m(u) .
$$

Предположим, что $t=t(u)>k$. В силу следствия 2 вектор $y=u[t]$ является максимальным ОВ. Поэтому

$$
\begin{aligned}
p+n-q & =|\operatorname{ind}(y)|=|\operatorname{ind}(u)|-|\operatorname{ind}(u, t)|-1+n-t \\
& >|\operatorname{ind}(u)|-(m(u)-t)-1+n-t=p+n-q,
\end{aligned}
$$

что невозможно.

Предположим теперь, что $m(u)>q$. Пусть $s-$ максимальный элемент в множестве ind $(b) \cap \operatorname{ind}(b \oplus x)$. Очевидно, что $s=m(u)$. Поэтому существует элемент $r \in \operatorname{ind}(b) \cap \operatorname{ind}(b \oplus x)$ такой, что $r \leqslant q$, и вектор $y=b-e_{s}+e_{r}$ является ОВ. Но тогда $(n-q-1)$-я производная вектора $y$ является левым сдвигом $\Phi(a)$, то есть является РВ. Получаем противоречие. 
Докажем теперь достаточность. Пусть $x$ - произвольный максимальный ОВ из сегмента $[v, w)$. Тогда $x=u[s]$ для некоторого $q \geqslant s>k \geqslant t(u)$ в силу следствия 2 . Из определения $t(u)$ следует, что $\operatorname{ind}(u, s)=m(u)-s$, откуда

$$
\begin{aligned}
|\operatorname{ind}(x)| & =|\operatorname{ind}(u)|-|\operatorname{ind}(u, s)|-1+u-s \\
& =|\operatorname{ind}(u)|-m(u)-1+n=p-q+n=|\operatorname{ind}(b)| .
\end{aligned}
$$

Кроме того, $\{q+1, \ldots, n\} \subseteq \operatorname{ind}(x)$.

Пусть теперь $y$ - произвольный максимальный ОВ из некоторого сегмента $\left[v_{1}, w_{1}\right)$, где $v_{1}, w_{1}$ - последовательные векторы из $\Omega^{*}$ (возможно, $\left.v=v_{1}, w+w_{1}\right), u_{1}=\Phi\left(v_{1}\right)$, $k_{1}=j\left(v_{1} \oplus w_{1}\right)$. Тогда $y=u_{1}\left[s_{1}\right]$ для некоторого $q \geqslant s_{1}>k_{1} \geqslant t\left(u_{1}\right)$ и $|\operatorname{ind}(y)|=$ $|\operatorname{ind}(b)|,\{q+1, \ldots, n\} \subseteq \operatorname{ind}(y)$.

Пусть $i \in \operatorname{ind}(x) \cap \operatorname{ind}(x \oplus y), j \in \operatorname{ind}(y) \cap \operatorname{ind}(x \oplus y)$. Тогда по определению замыкания $i \leqslant q, j \leqslant q$. Кроме того, ind $(b)=\{1, \ldots, p, q+1, \ldots, n\}$. Поэтому вектор $x-e_{i}+e_{j}$ является (впрочем, как и векторы $x, y$ ) правым сдвигом $b$, то есть является ОВ. Достаточность следует теперь из леммы 1.

Следствие 7. Регулярная $(p+1)$-униформная $(p \geqslant 1)$ система $[T, \mathscr{P}]$ является матроидом тогда и толъко тогда, когда ЯР является реберным множеством полного гиперграфа с множеством вершин $P_{1} \cup \ldots \cup P_{m}$.

Доказательство. Достаточность очевидна. Докажем необходимость. По условию для любого $v \in \Omega^{*}$

$$
|\operatorname{ind}(\Phi(v))|=|\operatorname{ind}(\Phi(a))|=p+1,
$$

откуда, $q=m(\Phi(v))$ согласно следствию 6 . Осталось показать, что любой вектор $x$, для которого $|\operatorname{ind}(x)|=p$ и $m(x)<q$, принадлежит $\Omega^{*}$. Предположим противное и выберем наименьший относительно порядка « вектор $x$ с такими свойствами, не принадлежащий $\Omega^{*}$. Пусть $s=\max \left\{i: x_{i}=0, \operatorname{ind}(x, i)=\{i+1, \ldots, m(x)\}\right\}, l=$ $m(x)-s-1$. Обозначим $y$ такой вектор, что $j(x \oplus y)=s$ и

$$
\operatorname{ind}(y, s)=\{q-l, q-l+1, \ldots, q-1\} .
$$

Очевидно, что $t(\Phi(y))=s$. Так как $y \ll x$, то $y \in \Omega^{*}$. Если теперь $z$ - вектор из $\Omega^{*}$, следующий за $y$ и $k=j(y \oplus z)$, то по следствию $6 s \leqslant k$. В доказательстве следствия 2 показано, что $y_{k}=1, z_{k}=0, z_{k+1}=\ldots=z_{m(z)}=1$. Отсюда, с учетом того, что $|\operatorname{ind}(z)|=p$, получаем, что $k=s$, и, следовательно, $z=x$. Получаем противоречие.

\section{4. Униформные бинарные системы}

В этой главе все рассматриваемые системы считаются униформными. Приведем вначале несколько определений. Для $n$-вектора $x$ назовем $\{i, j\}$-проекцией (или, эквивалентно, $\{j, i\}$-проекцией) $(n-2)$-вектор $x\{i, j\}$, полученный из $x$ исключением $i$-й и $j$-й компонент.

Введем обозначение $\Omega(\overline{k, l})=\left\{x: x \in \Omega, x_{k}=1, x_{l}=0\right\}$. Тогда $\Omega(k, l)$. будет обозначать множество $\{k, l\}$-проекций всех векторов из $\Omega(\overline{k, l})$.

Скажем, что система $\left[T, \Omega^{\prime}\right]$ получается из $[T, \Omega]$ преобразованием сдвига $(k, l, \Omega)$, если каждый вектор $u$ из $\Omega(\overline{k, l})$ такой, что $u\{k, l\} \notin \Omega(k, l)$, заменяется вектором $u-e_{l}+e_{k}$. В этом случае мы пишем $\left[T, \Omega^{\prime}\right]=\operatorname{shift}(k, l, \Omega)$.

Определим порядок $\simeq$ на множестве $T$, полагая $k \simeq l$, если и только если $\Omega(l, k) \subseteq$ $\Omega(k, l)$. 
Лемма 2 ([10]). Система $[T, \Omega]$ регулярна тогда и только тогда, когда $1 \simeq \ldots \simeq n$.

Лемма 3. Пустъ $\left[T, \Omega^{\prime}\right]=\operatorname{shift}(k, l, \Omega), p_{k} \leqslant p_{l}$. Тогда $h_{1}=h([T, \Omega], \bar{p}) \geqslant h_{2}=$ $h\left(\left[T, \Omega^{\prime}\right], \bar{p}\right)$.

Доказательство. Определим отображение $g$, заданное на множестве ОВ системы $[T, \Omega]$, полагая $g(x)=x-e_{k}+e_{l}$, если $x_{l}=0$ и $z \leqslant x$ для некоторого вектора $z$ такого, что $z_{k}=1$ и $z-e_{k}+e_{l} \in \Omega$, и $g(x)=x$ в противном случае.

Докажем вначале, что $g$ - инъективное отображение и каждый вектор $g(x)$ является ОВ системы $\left[T, \Omega^{\prime}\right]$.

Пусть $x$ и $y$ являются ОВ системы $[T, \Omega]$, причем $x \neq y, g(x)=g(y)$. Тогда точно один из векторов $x, y$, скажем, $y$, неподвижен относительно $g$, то есть $g(y)=y$, $g(x)=x-e_{k}+e_{l}$. По определению $g$ существует вектор $z$ такой, что $z-e_{k}+e_{l} \leqslant$ $x-e_{k}+e_{l}=g(x)=g(y)=y$ и $z-e_{k}+e_{l} \in \Omega$. Отсюда следует, что $y$ есть РВ. Получаем противоречие.

Теперь предположим, что $g(x)$ является РВ системы $\left[T, \Omega^{\prime}\right]$. Другими словами, $g(x) \geqslant z$ для некоторого вектора $z$ из $\Omega^{\prime}$. Если $g(x)=x$, то $z \notin \Omega$, поскольку $x$ является ОВ системы $[T, \Omega]$ и $z \leqslant x$. Следовательно, $z \in \Omega^{\prime}(k, l)$ и $z-e_{k}+e_{l} \in \Omega$. А это, по определению $g$, означает, что $x_{l}=1$. Но тогда $z-e_{k}+e_{l} \leqslant x$, то есть $x$ является $\mathrm{PB}$, что невозможно. Пусть $g(x)=x-e_{k}+e_{l}$. Так как $z_{k}=0$, то $z \in \Omega \cap \Omega^{\prime}$. Кроме того, $z_{l}=1$, ибо иначе $z \leqslant x$. Следовательно, $z \in \Omega(\overline{l, k})$ и существует $y$ из $\Omega(\overline{l, k})$ такой, что $y\{k, l\}=z\{k, l\}$. Но тогда $y \leqslant x$ и $x$ является РВ. Получаем противоречие.

Из предыдущих свойств отображения $g$ следует, что

$$
1-h_{2}=\sum_{f^{\prime}(y)=0} p(y) \geqslant \sum_{f(x)=0} p(g(x)) \geqslant \sum_{f(x)=0} p(x)=1-h_{1} .
$$

Последнее неравенство следует из очевидных соотношений

$$
p(g(x))= \begin{cases}p(x), & \text { если } x=g(x), \\ p(x) p_{l}\left(1-p_{k}\right) /\left(p_{k}\left(1-p_{l}\right)\right), & \text { если } x \neq g(\dot{x}) .\end{cases}
$$

Из свойств отображения $g$, полученных при доказательстве леммы 3 , вытекает следующее утверждение.

Лемма 4. Пусть $\left[T, \Omega^{\prime}\right]=\operatorname{shift}(k, l, \Omega)$. Тогда коэффициенты полинома надежности системы $\left[T, \Omega^{\prime}\right]$ не превосходят соответствующих коэффициентов полинома надежности системы $[T, \Omega]$.

Лемма 5. Пусть $\left[T, \Omega^{\prime}\right]=\operatorname{shift}(r, l, \Omega) u r \gtrsim s$ в $\left[T, \Omega^{\prime}\right]$. Tогда $r \gtrsim s$ в $\left[T, \Omega^{\prime}\right]$.

Доказательство. По определению преобразования сдвига, $\Omega^{\prime}(\overline{s, r}) \subseteq \Omega(\overline{s, r}), \Omega(\bar{r}, \bar{s}) \subseteq$ $\Omega^{\prime}(\overline{r, s})$. По определению порядка $\gtrsim, \Omega(s, r) \subseteq \Omega(r, s)$. Ввиду предшествующих включений $\Omega^{\prime}(r, s) \subset \Omega^{\prime}(r, s)$, а это означает, что $r \gtrsim s$ в $\left[T, \Omega^{\prime}\right]$.

Лемма 6. Пусть $\left[T, \Omega^{\prime}\right]=\operatorname{shift}(s, l, \Omega) u r \gtrsim s, r \gtrsim l$ в $[T, \Omega]$. Тогда те же соотношения между $r$, s u l справедливы и в $\left[T, \Omega^{\prime}\right]$. 
Доказательство. Вначале выберем вектор $x$ из множества $\Omega^{\prime}(\overline{s, r}) \backslash \Omega(\overline{s, r})$. Такой выбор $x$ означает, что $y=x-e_{s}+e_{l} \in \Omega(\overline{l, r})$ и $y_{r}=0$. Так как $r \gtrsim l$ в $[T, \Omega]$, существует вектор $z \in \Omega(\overline{r, l})$ такой, что $z\{r, l\}=y\{r, l\}$. Отсюда следует, что $z_{s}=0$, $z \in \Omega^{\prime}(r, s)$ и $z\{r, s\}=x\{r, s\}$. Итак, доказано, что $x\{r, s\} \in \Omega^{\prime}(r, s)$.

Теперь выберем вектор $x$ из множества $\Omega(\overline{s, r})$. Поскольку $r \gtrsim s$ в $[T, \Omega]$, существует вектор $y \in \Omega(\overline{r, s})$ такой, что $x\{r, s\}=y\{r, s\}$. Если $y \in \Omega^{\prime}$, то $x\{r, s\} \in \Omega^{\prime}(r, s)$. Пусть $y \notin \Omega^{\prime}$. Это выполняется, только если $y_{l}=1$. Следовательно, $x_{l}=1$. Так как $r \gtrsim l$ в $[T, \Omega]$, существует вектор $z \in \Omega(\overline{r, l})$ такой, что $z\{r, l\}=x\{r, l\}$. Но в этом случае $z \in \Omega(\overline{s, l})$ и $y\{s, l\}=z\{s, l\}$. Отсюда следует, что $y \in \Omega^{\prime}$, получаем противоречие.

Таким образом, доказано, что $x\{r, s\} \in \Omega^{\prime}(r, s)$ для каждого $x$ из $\Omega^{\prime}(\overline{s, r})$. Поэтому $r \gtrsim s$ в $\left[T, \Omega^{\prime}\right]$.

Поскольку $r \gtrsim s$ и $s \gtrsim l$ в $\left[T, \Omega^{\prime}\right]$, ввиду транзитивности порядка, $r \gtrsim l$ в $\left[T, \Omega^{\prime}\right]$. Лемма доказана.

Лемма 7. Пустъ $\left[T, \Omega^{\prime}\right]=\operatorname{shift}(k, l, \Omega)$ u $r, s, k, l$ различны $и r \gtrsim s$ в $[T, \Omega]$. Тогда $r \gtrsim s$ в $\left[T, \Omega^{\prime}\right]$.

Доказательство. Выберем произвольный вектор $x$ в $\Omega^{\prime}(\overline{s, r})$. Предположим вначале, что $x \notin \Omega$. Это выполняется, только если $u=x-e_{k}+e_{l}$ содержится в $\Omega(\overline{s, r})$. Поскольку $r \gtrsim s$ в $[T, \Omega]$, существует вектор $y$ в $\Omega(\overline{r, s})$ такой, что $y\{r, s\}=u\{r, s\}$. Если $y \notin \Omega^{\prime}$, то $w=y-e_{l}+e_{k} \in \Omega^{\prime}(r, s)$ и $w\{r, s\}=x\{r, s\}$. Если же $y \in \Omega^{\prime}$, то существует вектор $z$ в $\Omega(\overline{k, l})$ такой, что $z\{k, l\}=y\{k, l\}$. Но $z \in \Omega^{\prime}$ и $x\{r, s\}=z\{r, s\}$. Таким образом, в любом случае $x\{r, s\} \in \Omega^{\prime}(r, s)$.

Теперь предположим, что $x \in \Omega$. Так как $r \gtrsim s$ в $[T, \Omega]$, существует вектор $y$ в $\Omega(\overline{r, s})$ такой, что $y\{r, s\}+x\{r, s\}$. Если $x_{k}=1$ или $x_{k}=x_{l}=0$, то $x, y \in \Omega^{\prime}$ и снова $x\{r, s\} \in \Omega^{\prime}(\overline{r, s})$. Пусть $x_{k}=0$ и $x_{l}=1$. Так как $x \in \Omega^{\prime} \cap \Omega$, существует вектор $z$ в $\Omega(\overline{k, l})$ такой, что $z\{k, l\}=x\{k, l\}$. Отсюда следует, что $z \in \Omega(\overline{k, l})$. Последнее включение означает существование вектора $u$ в $\Omega(\overline{r, s})$ такого, что $u\{r, s\}=z\{r, s\}$. Но в этом случае $u \in \Omega(\overline{k, l})$ и $u\{k, l\}=y\{k, l\}$, то есть $y \in \Omega^{\prime}$. В результате получаем, что справедливо включение $x\{r, s\} \in \Omega^{\prime}(r, s)$.

В силу произвольности выбора $x$ заключаем, что $r \gtrsim s$ в $\left[T, \Omega^{\prime}\right]$. Лемма доказана.

Алгоритм R-SHIFT. Шаг 1. Переупорядочить элементы множества $T$ униформной системы $[T, \Omega]$ так, что $p_{1} \leqslant \ldots \leqslant p_{n}$.

War 2. for $i=1,2, \ldots, n-1$ do

for $j=i+1, \ldots, n$ do $\left[T, \Omega^{\prime}\right]:=\operatorname{shift}(i, j, \Omega) ;[T, \Omega]:=\left[T, \Omega^{\prime}\right]$; $\left[T, \Omega^{\prime}\right]:=[T, \Omega]$.

Из лемм 2-7 вытекает следующее утверждение.

Tеорема 2. Алгоритм R-SHIFT за полиномиальное времл строит регулярную систему $\left[T, \Omega^{\prime}\right]$, на которой достигаются нижние оценки надежности и коэффициентов полинома нажежности исходной системы $[T, \Omega]$.

\section{Список литературы}

1. Ball M. O., Provan J. Disjoint products and efficient computation of reliability. Oper. Res. (1988) 36, 703-715. 
2. Chernyak A. A., Chernyak Zh. A. Note on complexity of computing the domination of binary systems. Discrete Appl. Math. (1997) 73, 289-295.

3. Ball M. O., Provan J., Shier D. R. Reliability covering problems. Networks (1991) 21, 345357.

4. Colbourn C. J. The Combinatorics of Network Reliability. Oxford Univ. Press, New York, 1987.

5. Chari M. K., Provan J. Calculating $k$-connectedness reliability using Steiner bounds. Math. Oper. Res. (1996) 21, 905-921.

6. Ball M. O., Provan J. Bounds on the reliability polynomial for shellable undependence systems. SIAM J. Algebr. Discrete Math. (1981) 3, 166-181.

7. Hammer P. L., Johnson E. L., Peled U. N. Facets of regular 0-1 polytopes. Math. Programming (1975) 8, 179-206.

8. Wolsey L. A. Faces for a linear inequality of 0-1 variables. Math. Programming (1975) 8, 165-178.

9. Giles R., Kannan R. A characterization of threshold matriods. Discrete Math. (1980) 30, 181-184.

10. Peled U. N., Simeone B. Polynomial-time algorithms for regular set-covering and threshold synthesis. Discrete Appl. Math. (1985) 12, 57-69.

11. Hammer P. L., Pel d U. N., Pollatschek M. A. An algorithm to dualize a regular switching function. IEEE Trans. Comput. (1979) bf 28, 238-243.

12. Brown J. I., Colbourn C. J., Devitt J. S. Network transformations and bounding network reliability. Networks (1993) 23, 1-17.

13. Kelmans A. On graphs with randomly deleted edges. Acta Math. Acad. Sci. Hung. (1981) 37, 77-88.

14. Satyanarayana A., Schoppman L., Suffel C. D. A reliability-improving graph transformation with applications to network reliability. Networks (1992) 22, 209--216.

15. Рейнголд Э., Нивергельт Ю., Део Н. Комбинаторные алгоритмы. Мир, Москва, 1980.

Статья поступила 20.12.1996. Переработанный вариант поступил 28.01.1998. 